\title{
ANÁLISIS DE POLÍTICAS PÚBLICAS PARA EL FOMENTO DE LA CREACIÓN DE EMPRESAS EN EL MUNICIPIO DE VALLEDUPAR
}

(1998-2005)

\author{
WILFRIDO RODRIGUEZ OROZCO* \\ YIMY GORDON HERNÁNDEZ**
}

\section{RESUMEN}

Actualmente, se reconoce la necesidad de evaluar las políticas públicas aplicadas, debido a que sus resultados generan una visión del éxito obtenido en el contexto económico y social, a partir de la gestión de sus ejecutores. Las políticas públicas formuladas por el Estado con el fin de propender por el desarrollo, los elementos que las componen -por ejemplo, el fomento de mipymes- y la adecuación de los marcos legales y jurídicos para la promoción económica local, son evidencia de la acción del gobierno y de los subgobiernos; no obstante, la implementación de dichas politicas es una tarea de trabajo conjunto entre el Estado mismo y las entidades competentes. Este artículo presenta los resultados del análisis de políticas y acciones públicas en relación con los mecanismos de fomento a la creación de empresas en el municipio de Valledupar, ubicado en la costa caribe colombiana. La hipótesis conjetura un alto grado de desarticulación y descoordinación, entre las entidades cuyas competencias giran en torno al tema y un enfoque asistencial de los programas implementados por el ente territorial; sin embargo, este trabajo intenta hacer visibles las distintas arquitecturas organizacionales y financieras que han sido estructuradas por el Estado para resolver la necesidad de garantizar una acción territorialmente coordinada de las politicas sectoriales nacionales entre los años 1998 y 2005. Se utiliza el mecanismo de evaluación de políticas públicas a nivel de impacto (Roth Deubel, 2002). La información fue recolectada a través de encuestas a una muestra de 351 personas y entrevistas con representantes de 25 instituciones (entidades públicas, privadas, colegios, universidades e instituciones financieras, entre otros).

Palabras Clave: Políticas Públicas, Creación de empresas, normas, leyes, nuevas

* Candidato a Magíster en Gestión de Empresas y Economía Social de la Universidad Internacional de Andalucía UNIA (España). Presidente de la Fundación Caribe en la Sociedad del Conocimiento (Colombia). Coordinador Proyectos de Investigación Universidad Popular del Cesar UPC (Colombia). Miembro del grupo de estudios e Investigaciones Socioeconómicas y Desarrollo Regional GEISEDER (Colciencias “B"). Correo electrónico: wro_18@yahoo.es.

${ }^{* *}$ Ingeniero Mecánico de la Universidad Tecnológica de Bolivar, Especialista en Gerencia de Negocios Internacionales de la Universidad Jorge Tadeo Lozano, miembro del Grupo de Investigación FACE-UPC.

117 
empresas, empresarialidad.

Clasificación JEL: H70, H75

\begin{abstract}
At the moment, it is necessary the evaluation of the applied public policies, because their results will generate a visualization of the achieved success in the economic and social context, from the point of view of the executive board. The public policies formulated by the State to pretend the development and the elements that compose them - for example, the promotion of mipymes- and the adjustment of the legal and legal marks for the local economic promotion, are evidence of the action of the government and sub-governments; However, the implementation of political public policies is a common task between the State and the competent organizations. This article presents the results of the analysis of policies and public actions in relation to the mechanisms of promotion to the creation of companies in the municipality of Valledupar, located in the Colombian Caribbean coast. The hypothesis surmise a high degree of dislodgment and lack of coordination between the organizations whose assignment is a welfare approach of the programs implemented by the local governments, nevertheless, this work tries to make visible different organizations and their financial architectures that have been planned by the State to solve the necessity to guarantee an action territorially coordinated of the national policies between years 1998 and 2005. The mechanism of evaluation of public policies at impact level is used (Roth Deubel:2002). The information was collected through surveys to a sample of 351 people and interviews with representatives of 25 institutions (public organizations, deprived, schools, financial universities and institutions, among others).
\end{abstract}

Key Words: Public policies, enterprises creation, norms, laws, new enterprises, entrepreneurship.

JEL Classification: $\mathrm{H70}, \mathrm{H} 75$ 


\section{INTRODUCCIÓN}

En la mayoría de los países de Latinoamérica, existe una percepción asistencial frente a las micro y pequeñas empresas, a pesar de su importancia numérica y su trascendencia desde el punto de vista de generación de empleo e ingresos, con lo cual carecemos de diseños territoriales de políticas de fomento para la creación de este tipo de empresas. Este panorama sugiere un reconocimiento exhaustivo de los factores endógenos de cada territorio, para adecuar las políticas a cada realidad particular. En ocasiones, las limitaciones en las estrategias para implementación de políticas públicas de creación de empleo, provienen de un diseño inadecuado impuesto desde instancias externas y con un enfoque esencialmente asistencial; pero otras veces surgen por la inadecuación de los marcos jurídicos de los propios países, que impiden la coparticipación de las administraciones públicas territoriales y el sector privado empresarial en la creación de instituciones mixtas de desarrollo económico local.

Este documento explora si durante los 8 años de referencia ha habido un impacto relevante en cuanto al fomento de la creación de empresas en el municipio de Valledupar ${ }^{1}$. Las preguntas de investigación tienen la intención de establecer el contraste entre las políticas públicas y la acción política (práctica), sus posibles divergencias, vacíos y convergencias. Además, analizar su continuidad y grado de involucramiento de las entidades competentes para la implementación de las acciones tendientes a generar un ambiente propicio para el establecimiento de nuevas empresas en la red local. Lo anterior, bajo el supuesto de que existen factores externos que condicionan las decisiones de crear empresas o convertirse en empresario (Gómez, Veciana y Urbano: 2004), es decir, debe existir un ambiente o entorno favorable propiciado por una política pública territorial que reduzca las limitaciones formales e informales para el emprendimiento y constituya la base de la empresarialidad ${ }^{2}$ del territorio.

\footnotetext{
${ }^{1}$ El municipio de Valledupar está ubicado al nororiente de Colombia y es la capital del departamento del Cesar. Actualmente, registra una población aproximada de 350.000 habitantes según datos del Departamento Administrativo Nacional de Estadística - DANE. 2 Según Santos Cumplido (2004) la empresarialidad es el conjunto de cualidades dinámicas de empresa y empresarios, que influyen en la configuración de un determinado tipo de estructura productiva y empresarial y, por tanto, en el crecimiento y desarrollo económico de cualquier territorio.
}

119 


\section{OBJETIVO}

El objetivo es analizar el impacto de las políticas públicas implementadas por entidades cuyas competencias giran en torno al fomento de la creación de empresas, en el período de 1998-2005, en el municipio de Valledupar.

El proceso implica Identificar, inventariar y analizar comparativamente las políticas de fomento a la creación de empresas a nivel nacional y local de acuerdo con el contexto económico mundial y elaborar un inventario de instituciones relacionadas con el fomento de la creación de empresas en Valledupar, sus competencias y servicios. Además, determinar el resultado de los programas implementados por dichas entidades y su continuidad, los vínculos establecidos entre ellas, los sistemas de información o herramientas utilizadas. Lo anterior, con el fin de formular y proponer programas y estrategias para contribuir a la generación de iniciativas de desarrollo económico local en el municipio para el mediano y largo plazo a través del fomento de creación de empresas.

\section{MATERIALES Y MÉTODOS}

Básicamente y de manera generalizada, la evaluación de políticas consiste en una comparación entre las metas y objetivos propuestos inicialmente, durante la fase de formulación de políticas públicas, con los resultados obtenidos una vez se concluye la implementación de las mismas, a través de acciones y programas determinados. Desde un punto de vista más técnico, la evaluación de las políticas públicas es la manera más efectiva para determinar si las respuestas del Estado frente a las situaciones consideradas como problemáticas en la sociedad, han sido adecuadas y si otorgan una solución, es decir, si la política pública aplicada fue exitosa o no. La evaluación de las políticas públicas se clasifica de seis maneras diferentes, es decir, es posible realizar una evaluación desde diferentes ángulos e involucrando variables diversas.

Atendiendo la concepción de Roth Deubel (2002), según la cual la evaluación de impacto es la más diciente y veraz de las evaluaciones, ya que a 
diferencia de las demás evaluaciones (de medios, resultados, eficiencia, satisfacción y participativa) que parten de una base cuantitativa, ésta involucra principalmente aspectos cualitativos. Medir el impacto es un proceso complejo y profundo que permite incluso identificar los cambios de comportamiento que generan las políticas públicas, y el grado de concientización de los individuos. La identificación del impacto permite incluso direccionar las políticas formuladas e implementadas y determinar la conveniencia de su continuidad.

La investigación fue exploratoria y descriptiva, dado que el fenómeno no había sido estudiado en el municipio. Se utilizó información primaria y secundaria. La primera se obtuvo a través de encuestas a una muestra de 351 personas y entrevistas con representantes de 25 instituciones (entidades públicas, privadas, colegios, universidades e instituciones financieras, entre otros). La información secundaria corresponde al marco legal para fomento de la creación de empresas en Colombia, planes de acción municipales e informes de gestión municipales y entidades de apoyo.

\section{MARCO TEÓRICO}

\section{LAS POLÍTICAS PÚBLICAS COMO HERRAMIENTA DEL DESARRO- LLO ECONÓMICO LOCAL}

Muller y Surel (1998) consideran que una política pública "designa el proceso mediante el cual se elaboran e implementan programas de acción pública, es decir, dispositivos político administrativos coordinados en principio, alrededor de objetivos explícitos". El análisis de políticas públicas es valioso porque contribuye a la toma de decisiones, ofreciendo información con base en investigaciones, revelando incongruencias en los fines o en los procedimientos, generando nuevas alternativas y sugiriendo las maneras de trasladar las ideas políticas en políticas factibles y viables.

La urgente necesidad de iniciativas de desarrollo económico local se explica como resultado de un conjunto complejo de factores surgidos en los territorios destacando fundamentalmente los valores territoriales de identidad, diversidad y flexibilidad. Lo anterior, sumado al avance de los procesos de democratización y descentralización, con el consiguiente incremento de las funciones de las administraciones públicas territoriales, han obligado a los 
responsables políticos y técnicos a buscar enfoques y planteamientos apropiados para enfrentar los problemas y demandas crecientes de la población.

El aumento de la responsabilidad de las administraciones locales en el desarrollo económico y el empleo productivo ha supuesto una redistribución de las competencias y funciones, en materia económica, entre los diferentes niveles territoriales de las administraciones públicas. El impulso de la cooperación público-privada y la concertación estratégica de los actores socioeconómicos territoriales para diseñar estrategias locales de desarrollo local, han supuesto la aplicación de una gestión compartida para transformar sus sistemas productivos. Este objetivo, sin embargo, choca con la existencia de territorios desigualmente preparados para enfrentar tal desafío, lo que sugiere distintos tipos de intervención en términos de políticas públicas locales y regionales tendientes a mejorar sus capacidades competitivas. (Alburquerque, 2004; Alburquerque, 2007; Silva, 2005; Rui de Britto, 2000)

Para finalizar y con el propósito de establecer una conclusión acerca del desarrollo local y su relación con las políticas públicas, según Pinto (2006), anotaremos lo siguiente:

"Una iniciativa exitosa de desarrollo económico local se cimenta tanto en la motivación de individuos para ejecutar proyectos, como en la voluntad y habilidad de muchos de ellos para formular una visión compartida que guie los proyectos (...) Proponer proyectos cuyos actores locales no los consideran plausibles, no tiene ningun sentido. Tampoco tiene sentido proponer proyectos en los que se involucran actores que no se imaginan a ellos mismos trabajando juntos. Por tanto, se debe obtener no solamente un diagnóstico de las potencialidades económicas y sus retos, sino además, una idea clara sobre la capacidad, motivación y espíritu cooperativo de los actores claves".

\section{NORMAS ESPECÍFICAS Y DECRETOS}

En cuanto se refiere al marco jurídico, es decir, las políticas públicas generales puestas a disposición por el Estado para las regiones, y vigentes en el período de estudio, se destacan las siguientes leyes y decretos relacionados con el fomento a la creación de empresas en Colombia:

Ley 590 del 10 de julio de 2000. (Ley Mipyme) Esta es la ley marco de la política estatal de fomento a la creación de empresas en Colombia. Su objeto es "inducir el establecimiento de mejores condiciones del entorno institucional 
para la creación y operación de micro, pequeñas y medianas empresas". Además, obliga a los municipios y departamentos a establecer regímenes impositivos especiales con el fin de estimular la creación y fortalecimiento de las mipymes, y la reducción de aportes parafiscales por la creación de mipymes.

Ley 29 de 1990. (Ley de Ciencia y Tecnología) "Por la cual el Congreso de la Republica de Colombia dicta disposiciones para el fomento de la ciencia y tecnología". Esta ley es reglamentada por los decretos 393, 591 y 585 de 1991, que señalan las modalidades de asociación entre el Estado y particulares para el desarrollo de actividades científicas y tecnológicas, proyectos de investigación y creación de tecnología.

Decreto 1100 de 1992. "Por el cual el Congreso de la Republica de Colombia dicta disposiciones para promover el desarrollo de las Cooperativas empresariales".

Ley 344 de 1996. (Ley de Racionalización del Gasto Público) "Por la cual el Congreso de la Republica de Colombia dicta disposiciones para fomentar la innovación y el desarrollo tecnológico". Esta ley obliga al SENA a destinar el 20\% de sus ingresos para el desarrollo de programas de competitividad y desarrollo productivo, además, se crea el Fondo Emprender.

Ley 454 de 1998. "Por la cual el Congreso de la Republica de Colombia determina el marco conceptual que regula las empresas de Economía Solidaria, se transforma el Departamento Administrativo Nacional de Cooperativas en el Departamento Nacional de la Economía Solidaria, se crea la Superintendencia de la Economía Solidaria, se crea el Fondo de Garantías para las Cooperativas Financieras y de Ahorro y Crédito, se dictan normas sobre la actividad financiera de las entidades de naturaleza cooperativa y se expiden otras disposiciones".

Ley 795 de 2003. "Por la cual el Congreso de la Republica de Colombia ajusta algunas normas del estatuto orgánico del sistema financiero y se dictan otras disposiciones".

Ley 905 de 2004. "Por medio de la cual el Congreso de la Republica de Colombia modifica la Ley 590 de 2000 sobre la promoción del desarrollo de las micro, pequeña y mediana empresa colombiana".

Decreto 898 de 2002. "Por el cual el Congreso de la Republica de Colombia dicta el Código del Comercio. Regula la dirección, competencias y funcionamiento de las Cámaras de Comercio". 
Ley 1014 de 2006. (Ley de Fomento a la Cultura de Emprendimiento). Esta ley tiene como objeto "Promover el espíritu emprendedor en todos los estamentos educativos del país ". Esta última se relaciona dado que la investigación indaga acerca del conocimiento de la comunidad general respecto del tema y es posible que algunas personas la reconocieran por ser la más reciente y la que obliga a las instituciones educativas formales, privadas o públicas, a definir un área de formación específica para el emprendimiento.

\section{LAS POLÍTICAS PÚBLICAS DE FOMENTO A LA CREACIÓN DE EM- PRESAS: TENDENCIA INTERNACIONAL.}

Las directrices estipuladas a nivel internacional con el objeto de fomentar la creación de empresas y el desarrollo económico local, convergen en el involucramiento de planes y programas enfocados a la generación de herramientas de apoyo financiero, asistencia técnica para empresas, desarrollo tecnológico, cualificación del recurso humano, exportación de bienes y servicios con alto valor agregado y asociatividad.

Desde el punto de vista financiero, los lineamientos se orientan a crear fondos de inversión de desarrollo de empresas y fomentar programas para la asignación de microcréditos, aplicando metodologías en las instituciones para la asignación de recursos como la de grupos solidarios, bancos comunales, préstamos individuales, uniones de crédito y fondos rotatorios y priorizar la financiación de inversiones (maquinaria, adecuación, etc.), capital de trabajo e insumos de producción y comercialización en el extranjero de pequeñas industrias ${ }^{3}$.

Con respecto a los servicios de asistencia técnica, las disposiciones se encaminan a mejorar la gestión de las mipymes utilizando las asesorías especializadas para solucionar problemas específicos vinculados con aspectos como el análisis de mercado, diseño de productos, rediseño de procesos productivos, control de la contaminación y diseño e implementación de los sistemas de información.

Para incentivar el desarrollo tecnológico y fortalecimiento empresarial, se promueve la creación de fondos especializados, centros y parques tecnoló-

3 Lacalle Calderón (2001) hace una amplia descripción de los microcréditos como instrumento de lucha contra la pobreza. 
gicos; fomentar la realización de proyectos de investigación y desarrollo de productos, incluyendo pruebas y prototipos; generar la financiación y promoción de infraestructura física, equipos, instalaciones y para el desarrollo tecnológico de las empresas y, además, realizar alianzas estratégicas y/o asociaciones entre empresas del mismo sector para el logro de misiones tecnológicas en entornos extranjeros y para la transferencia de información.

En relación con la cualificación de talento humano, se ha establecido la creación de organismos públicos descentralizados y especializados en brindar capacitación técnica y empresarial; fomentar en las instituciones educativas de carácter privado (universidades, institutos de capacitación técnica, tecnológica, etc.), la inserción de nuevos programas de capacitación pertinentes, acordes con las necesidades y requerimientos del mercado.

En concordancia con el fenómeno de globalización de la economía se elaboran unas directrices orientadas a la generación de exportaciones de bienes y servicios con alto valor agregado consistentes en la disposición de incentivos tributarios y arancelarios, que beneficien a las empresas exportadoras; crear y/o disponer instituciones de gobierno encargadas de coordinar, administrar y hacer seguimiento a los programas para el fomento a la exportación; al fomento para la conformación de gremios y asociaciones para promover las exportaciones y la promoción de ferias, exposiciones y misiones empresariales.

Por último, se determina propender por la realización de alianzas estratégicas entre entidades gubernamentales y privadas, con carácter descentralizado, con el fin de implementar las disposiciones políticas para el crecimiento y desarrollo empresarial; promover la creación y fortalecimiento de figuras asociativas para la organización y gestión empresarial de funciones, operaciones y comercio y el incremento del capital humano.

\section{HALLAZGOS: EL CASO DE VALLEDUPAR}

\section{ANÁLSIS COMPARATIVO DE LAS POLÍTICAS A NIVEL GUBER- NAMENTAL (1998-2005)}

El impacto esperado con la implementación de las políticas públicas en el período de 1998-2000 es el mismo para los tres niveles institucionales (municipal, departamental y nacional) ya que se observa que los objetivos 125 
planteados por los entes gubernamentales en los planes de desarrollo correspondientes convergen en el tema de desarrollo local y la creación de empresas. Todos los planes de desarrollo evidencian programas y estrategias de financiación, asistencia técnica a las empresas, de fomento al desarrollo tecnológico, brindar capacitación para cualificar el recurso humano. A nivel nacional se exponen además, programas para fomentar la exportación de bienes y servicios con alto valor agregado, fomento a la asociatividad y la generación de alianzas estratégicas para la articulación de empresas, academia y entes gubernamentales. Adicionalmente, a nivel departamental se exponen programas para generar asociatividad y cooperación entre entidades públicas y privadas. Sin embargo, en el municipio de Valledupar no se evidencian disposiciones políticas relacionadas con el fomento a las exportaciones, ni tampoco la propensión a la realización de acuerdos de asociatividad y el trabajo conjunto entre los distintos actores involucrados en el tema de creación de empresas.

\section{RESULTADOS DE LA IMPLEMENTACIÓN DE LAS POLÍTICAS (AC- CIÓN POLÍTICA)}

\section{ALCALDÍA MUNICIPAL}

$\checkmark \underline{1998-2000}$

En el Plan de Desarrollo Municipal 1998-2000 "Valledupar, compromiso de todos" se observa la presencia de planes, programas y/o estrategias que fomentan de manera directa el fortalecimiento y desarrollo empresarial, y son:

- Consolidar el proceso de diversificación de la base económica del municipio para contrarrestar problemas estructurales y la vulnerabilidad de la producción de bienes y servicios.

- Fomentar la generación de empleo, a través, de la creación de comités locales

- Lograr alianzas estratégicas entre el sector público y privado, solidario, la inversión regional, nacional y extranjera para dinamizar y diversificar la economía local, por medio, del fomento para creación de empresas generadoras de empleo.

- Promocionar y apalancar el empleo productivo

- Gestionar el acceso a las fuentes de financiación de los programas de proyectos de desarrollo social 
- Crear condiciones para avanzar en el proceso y afianzar una cultura empresarial.

- Promover la organización de las empresas de comercialización de productos con origen agropecuario.

Se implementaron acciones dirigidas al Desarrollo Empresarial, de las cuales reporta un cumplimiento del $100 \%$ de las metas:

- Alianzas Estratégica para el Desarrollo Empresarial

- Estudios sectoriales referentes a la Oferta y Demanda de Bienes y Servicios Locales, Regionales, Nacional e internacional;

- Asesorías y Consultorías a Microempresarios; Consultorios empresariales y Fomento y Apalancamiento Financiero.

No obstante, carecen de evidencias la existencia de comités locales y la creación de condiciones para afianzar una cultura empresarial.

\section{$\underline{2001-2003}$}

En el Plan de Desarrollo Municipal 2001-2003 “Valledupar competitiva y solidaria" se evidencian algunas iniciativas como:

- Diversificar la base económica del municipio a través de la creación de centros productivos, incubación de empresas, transferencia tecnológica, innovación, desarrollo de productos, desarrollo empresarial y agroindustrial.

- Consolidar alianzas con el sector productivo-académico para el desarrollo empresarial.

- Apoyar los procesos de organización, financiación y comercialización implementando esquemas asociativos para la competitividad, productividad, equidad y fortalecimiento de la cultura empresarial.

- Implementar infraestructura y mecanismos de apoyo para la generación de empleo.

- Diversificar y mejorar la base productiva municipal.

- Fortalecer los sistemas de información municipal, a través de la implementación y optimización de los sistemas de información.

- Potencializar los sectores para la promoción y desarrollo de la Zona Económica Especial de Exportación, ZEEE.

- Crear una corporación para el desarrollo empresarial

- Crear el cluster de ganadería. 
- Fortalecer los procesos productivos y artesanales con fines de exportación.

- Impulsar procesos de producción y certificación de productos orgánicos.

- Impulsar y fortalecer la explotación y transformación de minerales.

- Aplicar nuevas tecnologías para el desarrollo de los procesos de enseñanza y aprendizaje, el desarrollo empresarial y la formación en artes y oficios.

En el año 2001 se inició la puesta en marcha de los siguientes proyectos, acerca de los cuales comentamos su estado:

- Generación de Empleo Permanente y Sostenible, incluyendo proyectos como el de Joven Emprendedor y Emprendedor Universitario. Se establecieron convenios interinstitucionales con universidades. Dichos convenios no están en ejecución.

- Valledupar Competitiva, tomando la atención de los sectores de panadería y confecciones. Se plantea un cumplimiento del $70 \%$, sin embargo actualmente las asociaciones creadas en estos sectores no operan como tales.

- Proyecto de Fondo de Iniciativa Empresarial. No hay evidencias de su existencia.

- Incubadora de Empresa del Sector Musical. No existe esta incubadora.

- Centros Demostrativos y Productivos Sectoriales, tomando como meta la creación de (4) C.D.P. se observa el cumplimiento de un 50\% de la meta. Este porcentaje de cumplimiento corresponde a la realización de los estudios de preinversión, sin embargo, hoy los centros no existen.

- Proyecto de Fomento de la Cultura Empresarial y Asociatividad. La meta se obtuvo con la capacitación de 150 personas. Proyecto exclusivamente de capacitación.

- Fortalecimiento del Sector de los Dulces Típicos, en el que capacitaron a 30 dulceras tradicionales. Se institucionaliza la feria del dulce.

Así mismo, en el 2002 se evidencia una continuidad en los programas, planes y estrategias establecidas en el año anterior. El Plan de Acción hace énfasis en el desarrollo empresarial y el trabajo productivo, la iniciativa empresarial y la incubación de empresas, a través de la ejecución de actividades de capacitación y formación empresarial.

Las inversiones realizadas entre los años 2001 y 2003 equivalen al 60\% de los recursos disponibles totales. La inversión realizada en los programas de "Generación de empleo permanente y sostenible, desarrollo empresarial y 
fomento al trabajo productivo" fue equivalente al $11 \%$ de los recursos generales de inversión.

Además, no existen evidencias de la existencia de un cluster de la ganadería, no se desarrolló la ZEEE y la Unidad Educativa de Formación Empresarial UNEFE operó sólo hasta el 2003.

\section{$\checkmark \underline{\text { 2004-2005 }}$}

En el Plan de Desarrollo Municipal 2004-2007 "Proyecto de vida" se observan las siguientes estrategias:

- Promocionar la economía para el empleo a través, del incremento de cadenas productivas y el fortalecimiento de su competitividad.

- Promover la producción cultural como alternativa socialmente productiva.

- Mejorar la eficiencia institucional a través de los sistemas de información que permitan dar un seguimiento y coordinación a las acciones y planes en pro de la administración del municipio.

- Realizar misiones empresariales.

- Fomentar la creación y fortalecimiento de asociaciones productivas de los subsectores más dinámicos de la economía

- Consolidar alianzas con el sector productivo, académico y organizaciones.

- Promocionar el desarrollo integral del potencial minero local con incorporación de valor agregado en las bases de producción y comercialización.

- Sistematizar de los procesos administrativos y financieros de las pymes.

- Conformar e implementar una red de apoyo institucional y empresarial.

- Incrementar las capacidades técnicas de las Mipymes a través, de la capacitación de empresarios.

- Crear centros de desarrollo productivo.

- Propender por el apalancamiento financiero de mipymes.

- Fomentar la comercialización a través de ruedas de negocio.

- Crear de empresas con perfiles competitivos locales.

- Asesorar mipymes en el proceso de certificación de calidad.

- Apropiación del fondo rotatorio empresarial. 
- Capacitar y acompañar a las mipymes para su organización, fortalecimiento y comercialización.

- Creación de cadenas productivas agro-empresariales.

- Rediseñar los currículos que refieren ajustes de nuevas cátedras de estudio.

- Impulsar y acompañar la comercialización de productos artesanales de las diferentes etnias a nivel nacional como internacional.

- Ofrecer la infraestructura adecuada para el desarrollo de la competitividad de las unidades empresariales.

Para el año 2004, la alcaldía municipal inició la puesta en marcha de los siguientes proyectos:

- Consejo Municipal para el Desarrollo Económico y el Empleo. A la fecha no encontramos evidencias de su existencia y operación.

- Sistema de Información para la Competitividad. Pretendía la consolidación de 4 asociaciones productivas de los subsectores confecciones, ebanistería, metalmecánica, dulces. Debemos destacar que en la actualidad de estas asociaciones sólo una opera como tal.

En el año 2005, la alcaldía municipal ejecuto los siguientes proyectos:

- Cadenas productivas para la competitividad y la generación de empleo, evidenciando la continuidad de proyectos anteriores.

- Creación y puesta en marcha del Consejo Municipal para el Desarrollo Económico y el Empleo. Se ejecuto un 10\% las metas para la convocatoria y sensibilización, un $40 \%$ de la elaboración del plan de acción, se logro un $30 \%$ de las metas establecidas para la conformación del observatorio económico y del empleo y un $20 \%$ de seguimiento, monitoreo y alimentación del programa.

- Sistema de Información para la Competitividad. Según el informe del plan de acción se realizo la definición del marco conceptual (40\%), adquisición del software $(40 \%)$, registro de toda la información $(10 \%)$, Seguimiento, monitoreo y alimentación del programa (10\%). Al respecto no se ha divulgado su existencia, ni su utilidad.

- Impulso a la creación y/o fortalecimiento de 4 empresas productivas de economía mixta, públicas y/o asociativas comerciales o de servicios afines a los perfiles competitivos locales. No existe evidencia de su existencia.

Además, quedaron inconclusas acciones como la creación de centros de desarrollo productivo, fondo rotatorio empresarial, creación de la cadena 
agro empresarial, promoción de la producción cultural como alternativa socialmente productiva, infraestructura adecuada para aumentar la competitividad.

La inversión que se realizó en el municipio de Valledupar en los programas de "Cadenas productivas para la competitividad y la generación de empleo, desarrollo agropecuario sostenible (reactivación del sector) y alternativas económicas de gran potencial." alcanzó en el año 2004 los 5.894,870 millones de pesos y en 2005, 20.245,000 millones de pesos. Lo anterior denota que de los recursos destinados a la realización de inversiones, el 11\% corresponde los programas relacionados con el tema empresarial.

TABLA No. 1. INVERSIÓN MUNICIPAL VS. NÚMERO DE EMPRESAS CONSTITUIDAS Y LIQUIDADAS (CIFRAS EN MILES DE PESOS)

\begin{tabular}{|c|c|c|c|c|c|}
\hline \multirow[b]{2}{*}{ AÑos } & \multirow[t]{2}{*}{$\begin{array}{l}\text { RECURSOS FINANCIEROS } \\
\text { DISPONIBLES }\end{array}$} & \multicolumn{2}{|c|}{ RECURSOS PARA INVERSIÓN EN E- MUNICIPIO } & \multicolumn{2}{|c|}{$\begin{array}{c}\text { EMPRESAS CONSTITUIDAS Y } \\
\text { LIQUIDADAS }\end{array}$} \\
\hline & & TOTAL & $\begin{array}{c}\text { FORTALECIM IENTO EMPRESARIAL Y } \\
\text { CREACIÓN DE EMPRESAS }\end{array}$ & CONSTITUIDAS & LIQUIDADAS \\
\hline 1998 & $\$ 29.087 .540$ & $\$ 13.092 .638$ & \multirow{3}{*}{$\$ 11.102 .854$} & 204 & 52 \\
\hline 1999 & $\$ 34.060 .897$ & $\$ 16.843 .113$ & & 197 & 81 \\
\hline 2000 & $\$ 38.425 .990$ & $\$ 18.941 .975$ & & 233 & 40 \\
\hline 2001 & $\$ 50.792 .036$ & $\$ 27.723 .743$ & $\$ 3.594 .852$ & 262 & 83 \\
\hline 2002 & $\$ 58.254 .878$ & $\$ 34.997 .734$ & $\$ 3.892 .864$ & 252 & 76 \\
\hline 2003 & $\$ 62.297 .040$ & $\$ 39.262 .255$ & $\$ 3.253 .299$ & 232 & 64 \\
\hline 2004 & $\$ 139.200 .140$ & $\$ 118.200 .786$ & $\$ 5.894 .870$ & 209 & 70 \\
\hline 2005 & $\$ 153.443 .148$ & $\$ 128.949 .083$ & $\$ 20.245 .000$ & 177 & 59 \\
\hline
\end{tabular}

Fuente: Elaboración del autor con base en registros de la Cámara de Comercio y Gacetas oficiales de la Alcaldía Municipal de Valledupar.

\section{SERVICIO NACIONAL DE APRENDIZAJE - SENA}

Los servicios ofrecidos por el Servicio Nacional de Aprendizaje - SENA -, en su Unidad Multisectorial de Emprendimiento, estuvieron orientados a la identificación de ideas de negocio potenciales, disposición de herramientas como acceso a Internet y asesoría permanente, asesoría en la formulación de planes de negocios y establecimiento de mecanismos para facilitar el acceso a créditos a través de entidades.

Los resultados encontrados fueron algunos convenios como los formalizados con la Fundación Animar y Electrificadora del Caribe para fomentar la creación de empresas que no reflejan resultados halagadores. Un convenio con la Alcaldía municipal y la Universidad Popular del Cesar, firmado en el año de 1998 y concluido en el 2001. El objeto de dicho acuerdo era la creación de una red de apoyo empresarial, de la cual no se conoce su operación y resultados. 
Es importante mencionar que durante la primera convocatoria del Fondo Emprender se presentaron 3 proyectos en el Cesar, de los cuales se aprobó solo un proyecto para su financiación con un monto de 69 millones de pesos, en tanto, para la segunda convocatoria del Fondo Emprender, se presentaron 7 proyectos, de los cuales se aprobó la financiación de cuatro de ellos por valor de 266 millones de pesos.

Desde la dirección general se han promovido algunos programas en todas las regionales, incluyendo Regional Cesar, como Programa de Formación y Asesoría a Creadores de Empresa, FACE (1999), en el marco del Programa Nacional de Creación y Fortalecimiento de Incubadoras de Empresas del SENA; Asesoría y Capacitación en Gestión para el Desarrollo Empresarial (2001), con el fin de mejorar la competitividad de los sectores económicos; Desarrollo de Incubadoras de Empresas de base tecnológicas (2001). Se realizo una Integración con El Sistema Nacional de Innovación; Fomento para la Organización de las Cadenas Productivas más Dinámicas en los Departamentos (2001). Convenios para impulsar la competitividad de los sectores en lo referente a la oferta exportadora del país, la incubación de empresas y el fomento de innovación de desarrollo tecnológico; Desarrollo De Competencias en Emprendedores (2005). Alumnos formados bajo los lineamientos de emprendimiento y empresarismo. Se logro con el apoyo de unidades de emprendimiento la identificación de iniciativas innovadoras que permitieron la formación de nuevas empresas que incrementaron el número y la calidad de los puestos de trabajo del país; Búsqueda de Empresas Padrino o Promotoras para el Apoyo del Emprendimiento (2005), Especialmente empresas surgidas desde el SENA, con la capacidad de prestar servicios a cadenas productivas y clusters, según la dinámica regional. Sin embargo, en el municipio de Valledupar los resultados no son evidentes, excepto los relacionados con la formación en los cursos propios que involucran la cátedra de emprendimiento.

\section{INSTITUCIONES EDUCATIVAS (COLEGIOS - UNIVERSIDADES)}

De manera generalizada, en los colegios del municipio aún no se han implementado las disposiciones políticas referentes al fomento de la creación de empresas. Algunos están iniciando el proceso; sin embargo, ninguna de las acciones corresponde al período de 1998-2005. 
Los Colegios Pablo VI y Gimnasio del Norte están en un proceso de formulación de planes y programas a ejecutar, acorde a las determinaciones establecidas en la ley 1014 de 2006. Así mismo, colegios como el Francisco Molina Sánchez y Nacional Loperena trabajan sobre el tema de las competencias laborales. Las demás instituciones, incluidas en el estudio, Gimnasio del Saber, Colegio Milciades Cantillo, la Concentración San Joaquín e INSTPECAM argumentaron no conocer el marco jurídico para el fomento de la creación de empresa. Se observa que el $90 \%$ de la población estudiantil de $11^{\circ}$ grado (último nivel secundario) no conoce el marco jurídico dispuesto por las entidades gubernamentales para fomentar y regular la creación de empresas.

Las universidades iniciaron procesos de fomento a la creación de empresas en el año 2001 con la aparición de las cátedras de emprendimiento dirigidas exclusivamente a estudiantes de administración de empresas (Universidad Popular del Cesar -UPC, Universidad de San Martín, Universidad Nacional Abierta y a Distancia -UNAD, Universidad de Santander -UDES) e ingeniería industrial (UDES). Estas cátedras han derivado en la realización de jornadas de sensibilización y ferias empresariales. Por su parte, las unidades de emprendimiento aparecen en el año 2005 en la UDES, sin resultados evidentes a la fecha, y en el año 2004 se diseñó en la UPC el proyecto de acuerdo para la creación de una unidad de emprendimiento, empero a la fecha no ha sido aprobado por el consejo superior. No obstante, existe un acuerdo con el Fondo Emprender que le permite a quienes operan como unidad en la UPC participar en sus convocatorias. En el período de estudio no se registran empresas creadas a partir de la orientación de estas unidades.

El estudio determinó que tres cuartas partes de la población universitaria encuestada no conoce el marco jurídico dispuesto por los entes gubernamentales para promover y regular la creación de empresas, es decir, el $75 \%$.

\section{INSTITUCIONES FINANCIERAS}

De acuerdo con el reporte de bancarización ${ }^{4}$ emitido por ASOBANCARIA acerca de los servicios financieros ofertados a nivel nacional, departamental

${ }^{4}$ Reporte de Bancarización a julio de 2006 - Vicepresidencia Económica - Dirección de estudios y regulación financiera CIFIN - ASOBANCARIA. El ranking de bancarización es 133 
y local, se evidencia que el departamento del Cesar presenta un bajo ranking de bancarización. (Ver tabla $\mathrm{N}^{\mathrm{o}}$ 2).

medido como la relación entre el número de personas con acceso a productos financieros y la población total. 
TABLA No. 2. PORCENTAJE DE LA POBLACIÓN CON ACCESO A PRODUCTOS FINANCIEROS A NIVEL DEPARTAMENTAL

\begin{tabular}{|c|c|c|c|c|c|c|c|}
\hline \multicolumn{8}{|c|}{ PRODUCTOS } \\
\hline DEPARTAMENTO & $\begin{array}{l}\text { Cuenta } \\
\text { de } \\
\text { ahorro }\end{array}$ & $\begin{array}{l}\text { Cuenta } \\
\text { Corriente }\end{array}$ & $\begin{array}{c}\text { Cartera } \\
\text { Total }\end{array}$ & $\begin{array}{l}\text { Cartera de } \\
\text { Consumo }\end{array}$ & $\begin{array}{c}\text { Cartera de } \\
\text { Vivienda }\end{array}$ & Microcrédito & $\begin{array}{c}\text { Tarjeta de } \\
\text { Crédito }\end{array}$ \\
\hline ANTIOQUIA & $29,8 \%$ & $2,83 \%$ & $8,12 \%$ & $6,50 \%$ & $0,89 \%$ & $0,81 \%$ & $4,52 \%$ \\
\hline SANTANDER & $28,4 \%$ & $3,47 \%$ & $7,55 \%$ & $4,89 \%$ & $1,27 \%$ & $1,45 \%$ & $6,64 \%$ \\
\hline BOGOTA & $54,3 \%$ & $8,37 \%$ & $19,86 \%$ & $15,14 \%$ & $4,19 \%$ & $1,47 \%$ & $27,0 \%$ \\
\hline ATLANTICO & $25,6 \%$ & $3,12 \%$ & $5,77 \%$ & $4,77 \%$ & $0,72 \%$ & $0,40 \%$ & $9,11 \%$ \\
\hline BOLIVAR & $16,7 \%$ & $1,76 \%$ & $3,49 \%$ & $2,90 \%$ & $0,42 \%$ & $0,19 \%$ & $2,01 \%$ \\
\hline SUCRE & $13,9 \%$ & $1,00 \%$ & $2,71 \%$ & $1,80 \%$ & $0,27 \%$ & $0,54 \%$ & $1,32 \%$ \\
\hline MAGDALENA & $16,6 \%$ & $1,37 \%$ & $2,65 \%$ & $1,93 \%$ & $0,40 \%$ & $0,24 \%$ & $1,64 \%$ \\
\hline LA GUAJIRA & $13,3 \%$ & $1,12 \%$ & $1,52 \%$ & $1,26 \%$ & $0,07 \%$ & $0,13 \%$ & $1,37 \%$ \\
\hline CORDOBA & $13,5 \%$ & $1,30 \%$ & $2,58 \%$ & $1,74 \%$ & $0,20 \%$ & $0,51 \%$ & $1,37 \%$ \\
\hline CESAR & $20,8 \%$ & $1,57 \%$ & $3,05 \%$ & $1,95 \%$ & $0,53 \%$ & $0,48 \%$ & $1,95 \%$ \\
\hline
\end{tabular}

Fuente: Reporte de Bancarización a Julio de 2006. ASOBANCARIA

Sin embargo, debemos destacar que los niveles de bancarización a nivel nacional son muy bajos, especialmente en lo que a microcréditos se refiere, como lo muestra la siguiente tabla.

TABLA No. 3. NIVEL DE BANCARIZACIÓN NACIONAL

\begin{tabular}{|c|c|c|c|}
\hline PRODUCTO & PERSONAS & $\begin{array}{l}\text { PORCENTAJE DE LA } \\
\text { POBLACIÓN }\end{array}$ & EMPRESAS \\
\hline CUENTA DE AHORRO & 11.257 .480 & 27,15 & 167.157 \\
\hline CUENTA CORRIENTE & 1.340 .128 & 3,23 & 253.361 \\
\hline CARTERA TOTAL & 3.230 .992 & 7,79 & 87.867 \\
\hline CARTERA COMERCIAL & 290.323 & 0,7 & 75.137 \\
\hline CARTERA CONSUMO & 2.335 .399 & 5,63 & 15.620 \\
\hline CARTERA VIVIENDA & 543.536 & 1,31 & 878 \\
\hline MICROCRÉDITO & 482.836 & 1,16 & 2.337 \\
\hline TARJETA DE CRÉDITO & 2.774 .361 & 6,69 & 42.658 \\
\hline
\end{tabular}

El porcentaje de personas con acceso a microcréditos en el municipio de Valledupar es de $0,34 \%$, cifra ésta muy inferior a la de ciudades intermedias como Pasto (4,70\%), Tunja (3,66 \%), Bucaramanga (1,37\%), Armenia $(1,28 \%)$, Neiva $(0,89 \%)$, e incluso menor que otros municipios del Cesar como Río de Oro (1,14 \%), San Alberto (1,76\%), Pelaya (1,51 \%) y Aguachi- 
ca $(0,76 \%)$. Esta situación contrasta con la tendencia internacional de fomento a la asignación de microcréditos a los individuos con menor posibilidad de acceso a los recursos financieros ${ }^{5}$.

\section{GREMIOS}

La Federación Nacional de Comerciantes - FENALCO- actúa como órgano representativo de los comerciantes, establece sistemas de información y propende por la participación en eventos que generen fortalecimiento, desarrollo y expansión a nivel empresarial para sus afiliados. En tanto, La Asociación Colombiana de Pequeñas Industrias- ACOPI-, es una agremiación que reúne a las pequeñas y medianas industrias constituidas formalmente; es una organización que representa a los empresarios, orienta, propone y propende por el establecimiento de políticas que promuevan el fortalecimiento y evolución a nivel empresarial para sus asociados. Es importante mencionar que ACOPI a nivel local no presenta evidencias acerca de la implementación de los planes y programas acordes a sus competencias durante el período de estudio, debido a que la entidad, no logro una organización eficiente que le permitiera cumplir a cabalidad con sus funciones y servicios. Actualmente, ACOPI Valledupar se halla en un proceso de reestructuración.

Por su naturaleza, ninguno de estos gremios presta servicios que fomenten de manera directa la creación de empresas.

\section{OTRAS ENTIDADES DE APOYO}

El impacto de la Incubadora de Empresas INCUBARCESAR ha sido reducido. Se concentró, en el período de estudio, en brindar asesoría, identificar proyectos con potencial, pero con pobres resultados. Se conoce que durante el período1998-2005, se incubaron 7 proyectos empresariales. Pese a ello, no existen evidencias de registros de seguimiento a estos proyectos. La Cámara de Comercio de Valledupar opera de acuerdo a las funciones estipuladas en el código del Comercio. Conforme a la naturaleza de la institución, las actividades ejecutadas están dirigidas a empresas puestas en marcha, por tanto, la Cámara de Comercio no se involucra directamente con el fo-

5 Lacalle Calderón (2001) hace una amplia descripción de los microcréditos como instrumento de lucha contra la pobreza. 
mento para la creación de empresas, dado que ofrece servicios de asesorías, acompañamiento empresarial y asistencia técnica a las empresas constituidas y registradas formalmente para su fortalecimiento y desarrollo empresarial. No obstante, la entidad hace parte del comité departamental de FOMIPYME, el cual se debe encargar de orientar las políticas para el fomento de la creación y fortalecimiento empresarial.

El Programa de las Naciones Unidas para el Desarrollo PNUD, no tiene competencias específicas con respecto al tema de creación de empresa. Los planes ejecutados están dirigidos a empresas constituidas y, por tanto, la entidad no se involucra directamente con el fomento para la creación de empresas. Sin embargo, el PNUD seccional Valledupar, por ser una organización no gubernamental y dedicada a la investigación y desarrollo tecnológico de las pequeñas y medianas empresas hace parte del Sistema Nacional de MIPYMES.

\section{LA COMUNIDAD EN GENERAL}

Existen tres razones por las que el $89 \%$ de la población no conoce el marco jurídico dispuesto para la creación de empresas. El primer argumento del $50 \%$ de los encuestados, es que "ha escuchado el marco jurídico, pero no es claro el conocimiento con respecto a este". La segunda razón, proveniente del 39\% de la población, es el "desinterés por crear empresa", y la ultima, es el hecho de que "nunca había escuchado este tipo de normatividades", aplicado para el $13 \%$ restante.

En cuanto al grado de reconocimiento que presentan los entes y entidades cuyas competencias giran en torno al tema de creación de empresas se observa que el $81 \%$ de la población perteneciente a la comunidad en general conoce alguna entidad de este tipo. Con respecto al grado de involucramiento y participación de la comunidad en actividades y programas ejecutados por las entidades competentes para fomentar la creación de empresas, se obtiene que el 54\% del total de la comunidad objeto de esta investigación, nunca ha participado de algún tipo de actividad y/o programa de este tipo.

Además, la dificultad más relevante a la hora de crear empresas es el la obtención o acceso de recursos económicos y/o financieros, encontrando que el $79 \%$ de la población encuestada afirma que en su caso la principal dificultad a la hora de crear empresa es la falta de recursos económicos, el 59\% afirma que lo es la dificultad para acceder a créditos financieros, la falta de 
información se convierte en una dificultad según el 52\% de la comunidad, seguido de los altos costos de legalización según el 50\%, la falta de asesoría según el $46 \%$, luego, el temor a la formalidad con el $25 \%$ y por último, la ausencia del fomento a la asociatividad se convierte en un problema para el $1 \%$.

\section{INVOLUCRAMIENTO ENTRE ENTIDADES PÚBLICAS Y PRIVADAS}

La Alcadia municipal, el SENA, la incubadora de empresas INCUBARCESAR, y demás entidades de apoyo como el PNUD, la Cámara de Comercio y tres (3) de las cuatro (4) instituciones de educación superior estudiadas han realizado algún tipo de convenio con el objeto de obtener cooperación para el desarrollo de actividades relacionadas con el tema de creación de empresas. Dichos acuerdos han girado en torno a la creación de fondos de iniciativas empresariales; la realización de estudios e investigaciones de tipo sectorial; la creación y puesta en marcha de un centro de servicios sociales y productivos; transferencia técnica y tecnológica específicas; unidad de emprendimiento; asesorías y consultorías a microempresarios; el fomento de la cultura empresarial y asociatividad y; el apalancamiento financiero. Sin embargo, dichos convenios no han tenido continuidad en su ejecución.

Por su parte, sólo en tres (3) de las diez (10) instituciones de educación media vocacional objeto de investigación han realizado acuerdos con instituciones como el SENA y el gobierno municipal con el propósito de involucrar a los estudiantes en un contexto empresarial y forjar el desarrollo de incipientes competencias laborales.

Es importante resaltar que las entidades financieras y gremios como FENALCO y ACOPI no evidencian a la fecha la realización de alianzas o acuerdos de cooperación con otro tipo de entidades u organismos para el fomento de la creación de empresas.

No se presenta de manera contundente un vinculo entre la academia, el gobierno y las empresas lo cual interfiere en la formulación de propuestas, planes y en la realización de actividades que ejecutadas de manera conjunta

y constante que permitan obtener mejores resultados en lo referente a la creación de empresas. 


\section{SISTEMAS DE INFORMACIÓN}

Es importante aclarar que no se obtuvo información específica acerca del plan de acciones ejecutado en el año 2003 por el gobierno correspondiente, debido a que dicha información no se encontró dentro de los archivos que posee la alcaldía.

En lo referente al fomento empresarial, de acuerdo al plan de acción municipal correspondiente al año 2005, se propuso la implementación de un Sistema de Información para la Competitividad. No obstante, el existen evidencias de la operación del sistema.

En instituciones de gran relevancia como el SENA, no se maneja información cuantitativa a nivel municipal, ya que esta, se comunica en informes de resultados nacionales y/o regionales, no obstante, se garantiza conforme a las afirmaciones de los directivos de la seccional Valledupar, la aplicación de los programas y acciones.

A pesar de la existencia a nivel local, de 4 programas universitarios de pregrado en ingeniería de sistemas y un sinnúmero de programas técnicos, sólo en una de las instituciones de educación superior (UPC) se cuenta con información relacionada con las actividades de emprendimiento realizadas y los proyectos y planes de negocio formulados, ejecutados y/o puestos en marcha. En las demás instituciones de educación superior, no hubo disponibilidad de la información y por tanto, no se encontraron datos cuantitativos acerca de los resultados de la implementación de las políticas.

De manera similar, es posible afirmar que no existen sistemas de información al interior de las instituciones educativas con formación media vocacional acerca del tema microempresarial, sin embargo, sólo en la Institución Francisco Molina se ha tratado de implementar un banco de ideas de negocio.

De otra parte, la Federación Nacional de Comerciantes ha establecido sistemas de información útiles para el fortalecimiento, desarrollo y expansión a nivel empresarial para sus afiliados. Por otro lado, en ACOPI no se evidencia la implementación de sistemas de información acerca del parque microempresarial del municipio. 
La Cámara de Comercio es uno de los principales órganos consultivos con los que cuenta el municipio ya que tiene a cargo la elaboración de los registros públicos y de costumbre; se encarga suministrar información de tipo mercantil, comercial y publicarla, a través de su participación en la elaboración de documentos como el Informe de Coyuntura Económica RegionalICER. No obstante, sus sistemas de información consisten en la elaboración de algunos estudios sectoriales y en el manejo de bases de datos físicas principalmente y algunas electrónicas.

De forma generalizada se evidencia la necesidad de aplicar, estandarizar y mejorar los sistemas de información, con el fin de generar herramientas de apoyo que faciliten la identificación de problemáticas, realización de diagnósticos, la toma de decisiones, la formulación de políticas públicas y, el seguimiento de las acciones realizadas, con el propósito de disponer de herramientas útiles para el fomento de la creación de empresas.

\section{CONCLUSIONES}

En Colombia existen políticas públicas para propiciar acciones de fomento de creación de empresas a nivel municipal. Es poco lo que puede proponerse en materia de políticas debido a que el país cuenta con mecanismos de apoyo en casi todos los aspectos relacionados con la MIPYMES, el problema evidenciado es que los emprendedores y la comunidad en general no manejan la información pertinente y las entidades competentes no se articulan de manera eficiente al Sistema Nacional de MIPYMES para encaminar acciones a nivel local. La iniciativa local a nivel de formulación de políticas es nula. No se evidencian, por ejemplo, regímenes tributarios especiales o fondos de inversión para los nuevos empresarios.

El modelo de inversión de recursos del municipio para el fomento de creación de empresas ha fracasado: no hemos tenido continuidad en las políticas de fomento a nivel municipal. Es relevante observar que las metas establecidas en los planes de desarrollo tienen niveles muy bajos de cumplimiento, además, se han enfocado principalmente a proyectos de desarrollo y fortalecimiento empresarial y no en los proyectos relacionados con el fomento de la empresarialidad o de una cultura de emprendimiento. Entre 1998-2000, el fomento para la creación de empresa no fue el principal objetivo ya que se hizo énfasis en el progreso y fortalecimiento empresarial, se observa que para el período 2001-2003 se formulan las primeras acciones dirigidas al fomento directo para la creación de empresa; sin embargo, sólo en el último período (2004-2005), los lineamientos evidenciados en el Plan de Desarrollo son más 
claros, amplios y corresponden a la formulación e implementación de proyectos para fomentar la cultura de emprendimiento, el empresarismo y la creación de empresas con el fin de generar competitividad y desarrollo local. Se manifiesta entonces, un incremento coyuntural en el número planes y/o programas formulados y ejecutados en torno a esta temática. A pesar de lo anterior, los resultados no muestran el cumplimiento de las metas propuestas.

Alto nivel de desconocimiento de las políticas y programas de fomento a la creación de empresas (89\%). La comunidad general desconoce las medidas de apoyo a la creación de empresas en el país y el municipio. Similar situación se encontró en la comunidad universitaria $(75 \%)$ y los estudiantes de media vocacional $(90 \%)$.

La falta de información y la dificultad de acceso a recursos financieros son considerados como los problemas más importantes para crear empresas. Para la comunidad, los problemas o dificultades más serias al momento de crear empresas lo constituyen la falta de recursos económicos (79\%) y el difícil acceso a créditos financieros $(59 \%)$. De igual manera piensa la comunidad académica universitaria ( $68 \%$ y $52 \%$ respectivamente), y adicionalmente argumentan la falta de información (48\%). En tanto que los jóvenes de media vocacional resaltan que el problema más relevante es la falta de información $(63 \%)$ y falta de asesoría (54\%).

Las universidades no están haciendo un aporte relevante al proceso. Las instituciones de educación superior han implementado recientemente cátedras como "Desarrollo del Espíritu Empresarial" y "Creación de Empresas". Sin embargo, sólo en una de dichas instituciones existe formalmente una Unidad de Emprendimiento, lo que ha contribuido a la baja identificación, formulación y ejecución de planes e ideas de negocio.

Las instituciones poseen sistemas de información obsoletos. A nivel municipal, entidades como la Alcaldía, el SENA, la Cámara de Comercio, Universidades, presentan un avance poco significativo con respecto al diseño, implementación y uso de los sistemas de información, con el fin de generar herramientas de apoyo que faciliten la identificación de problemáticas, realización de diagnósticos, la toma de decisiones, la formulación de políticas públicas y el seguimiento de las acciones realizadas, con el propósito de disponer de herramientas útiles para el fomento de la creación de empresas. Además, se identificó en la gran mayoría de las entidades relacionadas 
con el tema un aislamiento casi total del uso de nuevas tecnologías de la información y las comunicaciones TIC's.

El grado de cooperación entre instituciones es muy bajo. Las variables que determinan el éxito de las políticas para el fomento de la creación de empresas en un municipio, no están relacionadas únicamente con el conocimiento que las entidades competentes tienen sobre ellas, la implementación y la continuidad de dichas disposiciones políticas, sino también, de una serie de aspectos claves tales como la articulación de los diferentes entes públicos y privados con el fin de realizar un trabajo conjunto que haga más eficiente la ejecución de los programas, actividades y servicios dispuestos para promover la creación y el desarrollo empresarial. Se observa que a nivel municipal se han efectuado algunos convenios puntuales de cooperación para lograr la articulación Gobierno-Universidad-Empresa; empero, se estima que es necesario promover el establecimiento acuerdos estratégicos, haciendo partícipes a los gremios, las instituciones de educación media vocacional y entidades financieras, garantizando una labor de seguimiento a sus acciones para evitar que los impactos sean asistenciales como ha venido sucediendo. El Consejo departamental de Mipymes, para citar otro ejemplo, no ha operado continuamente.

Los resultados de la implementación de las políticas públicas son el producto de la combinación y aplicación de las variables analizadas anteriormente. De manera generalizada, encontramos que en el municipio de Valledupar no se cuenta con datos específicos -a causa de la deficiencia en el diseño, implementación y estandarización de los sistemas de información-, acerca de los resultados obtenidos como consecuencia de la puesta en marcha de los planes de acción gubernamentales y de los diferentes programas y actividades llevados a cabo por las entidades competentes para fomentar la creación de empresas, lo que obstaculiza la implementación de un proceso de seguimiento y evaluación. Sin embargo, es importante señalar que dentro de los hallazgos de la investigación para el período de 1998-2005, se observa que el gobierno municipal ha dado sus primeros pasos en torno al tema de creación de empresas con la proposición de proyectos, no sólo de desarrollo y fortalecimiento empresarial, sino además, de aquellos relacionados con emprendimiento, incubación de empresas, fomento de la cultura empresarial y asociatividad.

\section{LÍNEAS DE TRABAJO SUGERIDAS}


Financiación: Promover y facilitar el acceso a la financiación de proyectos emprendedores y el fortalecimiento de la MIPYME local, a través de:

a) Programas para la asignación de microcréditos, aplicando metodologías en las instituciones para la asignación de recursos, como la de grupos solidarios, bancos comunales, préstamos individuales, uniones de crédito o fondos rotatorios.

b) Divulgar eficazmente los programas de subsidios y seguros de crédito orientados a solucionar la insuficiencia de garantías que presentan las mipymes

c) Priorizar la financiación de 4 tipos de proyectos: creación de empresas, modernización, cooperación competitiva e I+D+I.

Asistencia técnica: Mejorar la gestión organizacional y operacional de las empresas, brindando asesorías especializadas, como instrumento para solucionar problemas específicos vinculados con aspectos como los análisis de mercado, el diseño de productos, el rediseño de procesos productivos, el control de la contaminación y los sistemas de información. Podemos sugerir éstas acciones:

a) Promover la formalización de las empresas

b) Seguimiento y evaluación de las entidades competentes con el fin de establecer el cumplimiento de sus responsabilidades con respecto al fomento de la creación de empresas.

c) Establecer grupos de asesores (estudiantes) en universidades e instituciones afines.

d) Institucionalizar jornadas de sensibilización empresarial

e) Promover el desarrollo de industrias culturales en el municipio.

Desarrollo tecnológico: ampliar las capacidades productivas de la región y potencializar las ventajas que actualmente posee nuestro territorio a través del desarrollo, fabricación y comercialización de nuevos productos y servicios.

a) Incentivos económicos para atraer la instalación de pequeñas industrias en el parque industrial de Valledupar

b) Aumentar los indicadores de conectividad local

c) Puesta en marcha de los CDP's prioritarios

d) Crear fondos comunes especializados en promover, coordinar y ejecutar acciones financieras en pro del desarrollo tecnológico y productivo.

e) Crear vínculos universidad- empresa con el fin de promover proyectos de investigación y desarrollo de productos y servicios e incluyendo pruebas y prototipos.

f) Diseño e implementación de sistemas de información eficientes relacionados del parque microempresarial de Valledupar 
Capacitación: Generar una cultura de emprendimiento en la comunidad general del municipio y formar un recurso humano capaz de aprovechar las potencialidades del territorio agregando valor a sus productos y servicios.

a) Creación de un fondo común para la cualificación del talento humano del municipio

b) Utilización de medios alternativos de promoción de la cultura de emprendedora

c) Inclusión de cátedras de emprendimiento en el plan de estudio en Universidades e instituciones educativas.

Asociatividad: Promover a través de las figuras asociativas, la organización y gestión empresarial de funciones, operaciones y comercio; el incremento del capital humano debido a su formación y capacitación; el acceso a instrumentos y entidades tecnológicas, y beneficios por asesorías y acompañamiento empresarial, con el objeto de incrementar la productividad y competitividad. Este objetivo s puede apoyar con acciones como:

a) Promover el establecimiento de empresas de reinserción sociolaboral, empresas de economía social en la región

b) Puesta en marcha de la Agencia de Desarrollo Local (ADEL) como escenario de concertación para las unidades productivas y de servicios para llegar a acuerdos asociativos entre micros y pequeñas empresas con otras de mayor tamaño. 


\section{REFERENCIAS BIBLIOGRÁFICAS}

Alburquerque, F. (2004). Desarrollo Económico Local y Descentralización en América Latina. Revista de la CEPAL, 82, 167-171.

Alcaldía Municipal: Valledupar Competitiva y Solidaria. (2003). Informe De Gestión 2001-2003. Valledupar: Autores.

Asociación Nacional de Industriales [ANDI]-Organización Internacional de Trabajo [OTI]. (2006). Análisis y Propuestas: Estudio Sobre La Informalidad y Pymes en Colombia. Bogotá: Autores.

Banco de La Republica-Departamento Administrativo Nacional de Estadísticas [DANE]. (2006). Informe de Coyuntura Económica Regional [ICER]. Valledupar: Autores.

Congreso de la República de Colombia. (2000). Ley 590 del 10 de Julio de 2000. "Por la cual se dictan las disposiciones para promover el desarrollo de los micros, pequeñas y medianas empresas. Santa Fe de Bogotá.

Congreso de la República de Colombia. (2002). Ley 789 de 2002. "Por la cual se dictan normas para apoyar el empleo y ampliar la protección social y se modifican algunos artículos del Código Sustantivo de Trabajo.". Santa Fe de Bogotá.

Congreso de la República de Colombia. (2006). Ley 1014 del 26 de enero de 2006 "Por la cual se dictan las disposiciones de fomento a la cultura de emprendimiento". Santa Fe de Bogotá.

Congreso de la República de Colombia. (2006). Legislación Cooperativa: "Por la cual se actualiza la Legislación Cooperativa". Momo Ediciones.

Fernández, J. (2005). Empresarialidad y Evolución Económica a Largo Plazo: La Relación De Empresarialidad y Evolución Económica a Largo Plazo: Universidad de Sevilla; Facultad de Ciencias Económicas y Empresariales: Departamento de Economía Aplicada I. 
Gaviria, J. (2006). De Empresas y Empresarios: Reflexión En Torno Al Sentido Y Significado De Estos Conceptos Desde La Etimología Y Desde La Teoría Económica. Semestre Económico, 9/17, 127-155.

Gómez, L., Martínez, J., Arzuza, M. (2006). Política Pública y Creación de Empresas en Colombia. Pensamiento y Gestión. N ${ }^{\circ} 21$. Universidad del Norte. Barranquilla.

Gómez, L., Veciana, J.M., Urbano, D. (2004). Medidas de Apoyo a la Creación de Empresas en el Caribe Colombiano. Investigación y Desarrollo. Vol. 12, No 2. Págs. 372-395. Universidad del Norte. Barranquilla.

Gutiérrez, J. (2005). Estructura del Mercado laboral Colombiano y Análisis de Coyuntura 2004 - 2005. Medellín: Universidad de Medellín, Centro de Investigaciones Económicas, Administrativas, Contables y Afines [CIECA].

Guzmán, S. (2006). Planificación y Gestión del Territorio: Una Propuesta de Desarrollo Endógeno para un Municipio Colombiano, caso Marsella- Risaralda. Medellín: Universidad de Medellín; Centro de Investigación en Ingeniería.

Guzmán J. \& Liñan F. (2005). Evolución de La Educación Empresarial en Estados Unidos y Europa: Su Papel como Instrumento de Desarrollo. Revista de Economía Mundial, 12, 149-171.

Lacalle Calderón, M. (2001). Los Microcréditos: Un Nuevo Instrumento de Financiación para Luchar contra la Pobreza. Revista De Economía Mundial, 5, 121-138.

Maussa, F; Tejada, M \& Rueda, O; (2004). La Competitividad en Sectores Fragmentados como las Artesanías en Bogotá y CundinamarcaColombia (Proyecto CINNCO). Bogota: Pontificia Universidad Javeriana.

Montoya, J. Anotaciones sobre la Historia Empresarial de Pereira. Risaralda: Universidad Católica de Risaralda. Grupo de Desarrollo Empresarial.

Muller, P. (2006). Las Políticas Públicas. Traducción. Universidad Externado de Colombia. $2^{\circ}$ Edición. Bogotá D.C. 
Oficina de Planeación Departamental (2001). Plan de Desarrollo Departamental 2001-2003: Compromiso con Nuestro Futuro. Valledupar.

Oficina de Planeación Departamental (2004). Plan de Desarrollo Departamental 2004-2007: Para los Buenos Tiempos. Valledupar.

Oficina de Planeación Departamental. (1998). Plan de Desarrollo Departamental 1998-2000: Hacia Un Nuevo Siglo, Un Cesar Para Todos. Valledupar.

Oficina de Planeación Municipal. (1998). Acuerdo No 015 del 27 de mayo de 1998. "Por el cual se adopta el Plan de Desarrollo Municipal de Valledupar 1998-2000: Valledupar, compromiso de todos". Valledupar.

Oficina de Planeación Municipal. (2001). Acuerdo Nº 002 del 30 de mayo de 2001. "Por el cual se adopta el Plan de Desarrollo Municipal de Valledupar 2001-2003: Valledupar, competitiva y solidaria". Valledupar.

Oficina de Planeación Municipal. (2004). Acuerdo $\mathrm{N}^{\circ} 011$ del 22 de mayo de 2004. "Por el cual se adopta el Plan de Desarrollo Municipal de Valledupar 2004-2007: Proyecto de Vida". Valledupar.

Organización para la Cooperación y El Desarrollo Económico [OCDE], (2003). Iniciativa Empresarial Y Desarrollo Económico Local: Recomendaciones Para La Aplicación De Programas Y Políticas. Paris: OCDE (Eds.).

Pinto, María Elisa (2006). Desarrollo Económico Local: Orientaciones E Instrumentos Para Alcaldes Y Gobernadores. Debate N ${ }^{\circ}$ 15.Bogotá, Colombia: Legis.

Roth Deubel, A. (2002). Políticas Públicas: Formulación, Implementación y Evaluación. Bogota, Colombia: Ediciones Aurora.

Rui de Britto Álvarez, A. (2000). Decentralizacao, desenvolvimento local e crisis da Federacao no Brasil. Santiago de Chile. CEPAL

Santos Cumplido, F. (2004). Convergencia, Desarrollo y Empresarialidad en el Proceso de Globalización Económica. Revista de Economía Mundial, 10/11, 171-202. 
Secretaria de Planeación Municipal. (2005). Anuario Estadístico Municipio de Valledupar 2005: Proyecto de Vida. Valledupar.

Silva, I. (2005). Desarrollo Económico Local y Competitividad Territorial en América Latina. Revista de la CEPAL, 85, 81-100.

Tafur, D. (2003). Políticas Públicas (Módulo de Especialización en Gerencia Pública).Valledupar: Universidad de Santander [UDES].

Yoguel, G. (2000). Creación de Competencias en Ambientes Locales y Redes Productivas. Revista de la CEPAL, 71,105-119.

Universidad Nacional De Colombia. Facultad de Derecho, Ciencias Políticas y Sociales. (2003). Formulación, Implementación, y Evaluación de Políticas Públicas. (Curso de Extensión). Ciudad: Autores. 